\title{
Towards 3D simulation of sintering processes
}

\author{
S. Bordère ${ }^{1}$, D. Bernard ${ }^{1}$, S. Vincent ${ }^{2}$ \& J.-P. Caltagirone ${ }^{2}$ \\ ${ }^{1}$ Institut de Chimie de la Matière Condensée de Bordeaux, \\ ICMCB-CNRS-Université Bordeaux 1, France \\ ${ }^{2}$ Transferts, Ecoulements, Fluides Energétique, \\ TREFLE-ENSCPB, France
}

\begin{abstract}
The Monte Carlo approach based on minimisation of a potential including interface and volume energies has been implemented in the framework of a Eulerian interface tracking method in order to deal with 3D sintering of complex powder compacts. First applied to the unique spherical particle system, these new developments lead to a surface tension-induced pressure close to that theoretically given by Laplace's law. The accuracy of this result is largely improved compared to those given by a classical fluid mechanics computation using the front tracking method. Our progress towards 3D modelling of sintering for realistic systems is illustrated by the computed stress gradients induced by surface curvature gradients within a small set of particles extracted from X-ray computed micro-tomography images of a real sample.

Keywords: stochastic methods, ceramics, image analysis.
\end{abstract}

\section{Introduction}

As regards the recent studies in modelling sintering processes [1-4], one can notice that it still remains difficult to deal with realistic particle arrangements systems. In fact, modelling of polycrystalline material sintering has only been handled in $2 \mathrm{D}[1,2]$. The only approach which would be powerful enough to overcome that difficulty is the phase field approach which is based on a diffuse definition of the interface [2]. Applied to 3D complex microstructure evolution only involving grain growth [5], no example of 3D many particle densification has up until now been shown. In the case of amorphous particles sintering, only $2[6,7]$ or 3 particles were handled [8]. Apart from these deterministic methods, the Monte Carlo simulations based on the Potts model have exhibited 3D 
microstructures evolutions [4] but remain too restrictive for precise morphology characterisation and realistic kinetics. The Monte Carlo methodology based on the non-discrete potential that we have developed was shown to overcome these restrictions [9-11]. Nevertheless, this methodology was only developed for 2D and $3 \mathrm{D}$ axi-symmetric configurations of particles, and therefore remains restrictive to deal with realistic arrangements of particles. Based on finite element discretisation, the main numerical difficulty to extend this approach to 3D modelling was to readapt the tetrahedral mesh during calculation. So, to progress towards 3D modelling of sintering, this Monte Carlo methodology is now implemented in the framework of a Eulerian interface tracking method. We present here, the first 3D results, with the aim to show that it is possible to model sintering processes involving real particle arrangements that can be extracted from microtomography images of powder compact.

\section{Simulation method}

\subsection{The Monte Carlo model}

The energetic model of the Monte Carlo methodology is defined in the canonical ensemble (closed system at constant volume and temperature) by the potential $\phi$ :

$$
\phi=\gamma_{s} A_{s}+E_{v}
$$

Here $\gamma_{\mathrm{s}}$ is the surface tension, $A_{s}$ its respective surface area and $E_{v}$ the total volume energy of the system which includes the volume energy of the solid phase and the vapor phase.

The volume energy in eq. (1) corresponds to the sum of two terms: $E_{v}=E_{0}+E_{\varepsilon}$, where $E_{0}$ is the volume energy for the two-phase coexistence involving no interface tension, and $E_{\varepsilon}$ is the excess of volume energy which is induced by an interface tension. This capillary induced excess of volume energy is quantified in the framework of elastic strain energy:

$$
E_{v}-E_{0}=E_{\varepsilon}=\frac{1}{2} \int_{V}(\sigma: \varepsilon) d V
$$

where $\varepsilon$ and $\sigma$ are the elastic strain and stress tensors, respectively. The strain tensor $\varepsilon$ is related to the displacement vector of the fluid phase, and the stress tensor $\sigma$ to the strain tensor through the elastic constants: the Young's modulus $E_{Y}$ and the Poisson's coefficient $v$. The minimization of the potential through the Metropolis algorithm allows the calculation of the stress gradient within the volume phase in relation to the surface curvature gradients. The hydrostatic pressure can thus be deduced from the relation:

$$
p=-\frac{1}{3} \operatorname{trace}(\sigma)
$$

This stress gradient is the driving force for volume mass transport which will allow the power compact to sinter. 


\subsection{The Monte Carlo implementation}

The solid/vapor interface is discretized using a triangular mesh (fig. 1(a)) for a Lagrangian description of the interface motion. This discretized interface is placed within a fixed Cartesian grid (fig. 1(a)) for a Eulerian description of the two volume phases. On this grid a staggered mesh is used (fig. 1(b)). In the following displacements of the volume phase will be imposed on the $u, v, w$-grids for the calculation of the strain and stress tensors on the $p$-grid (fig. 1(b)).

The $\phi$-potential is minimized using the Metropolis algorithm which proceeds in localized random changes of the total system configuration in order to induce potential variation $\delta \phi^{m}$ of the system. The configuration changes are accepted with the probability

$$
P\left(\delta \phi^{m}\right)=\min \left(1, \exp \left(-\delta \phi^{m} / k_{B} \mathrm{~T}\right)\right)
$$

where $k_{B}$ is the Boltzmann constant and $T$ the temperature.

Two types of random changes are considered (see [12] for a complete description of the method):

- the first one concerns configuration changes by local elastic displacement of the two-phase system. At step $m$, when displacement only involves the volume phase deformation, three points located respectively on the $u, v, w$-grids (fig. 1(b)) are randomly chosen. Then, random displacements $u^{m}, v^{m}, w^{m}$, at step $m$, are respectively imposed upon these three points. For each of them $(\mathrm{a}=$ $\mathrm{u}$ or $\mathrm{v}$ or $\mathrm{w}$ ), the variation of the strain tensor $\delta \varepsilon_{a, c}^{m}$ within the control volumes which are centered at the scalar points located backwards $(c=b)$ and forward $(c=f)$ can be calculated from the displacement vectors. The stress tensor $\delta \sigma_{a, c}^{m}$ can thus be deduced from elastic equation depending on the Young's modulus $E_{Y}$ and the Poisson's coefficient $v$.

At this stage the strain and stress tensors at step index $m+1$ can be calculated as: $\varepsilon^{m+1}=\varepsilon^{m}+\delta \varepsilon^{m}, \sigma^{m+1}=\sigma^{m}+\delta \sigma^{m}$.

Hence, the elastic strain energy variation $\delta E_{\varepsilon}^{m}$ which is determined from integration over the control volume $V_{a, c}^{m}$ can be determined using the following equation:

$$
\delta E_{\varepsilon}^{m}=\sum_{(a, c)=(u, b),(u, f),(v, b),(v, f),(w, b),(w, f)} V_{a, c}\left(\sigma_{a, c}^{m}+\frac{1}{2} \delta \sigma_{a, c}^{m}\right): \delta \varepsilon_{a, c}^{m}
$$

At step $m$, in the case of interface and volume displacement coupling, a Lagrangian marker $i$ is randomly selected among the $N_{f}$ interface markers (fig. 1b) and randomly displaced ( $\boldsymbol{u}_{f, i}^{m}$ is the corresponding displacement vector). This displacement vector is projected onto the Eulerian grid by choosing three points located in the vicinity of the $i$-marker on the $u$-grid, $v$ grid and $w$-grid respectively (fig. 1). These three markers support the fluid displacement values $u^{m}, v^{m}$ and $w^{m}$ along the $X, Y, Z$-directions respectively. 
That volume-displacement also implies the movement $\boldsymbol{u}_{f, l}^{m}$ of neighboring interface markers $l$ of the $i$-marker. Interface area $A^{m+1}$ at step $m+1$ can be determined from the new position of interface markers $\mathbf{x}_{f, a=(i, l)}^{m+1}=\mathbf{x}_{f, a=(i, l)}^{m}+\boldsymbol{u}_{f, a=(i, l)}^{E, m}$ allowing the calculation of the surface energy variation $\delta E_{s}^{m}$ at step $m$ induced by the initial $i$-marker displacement $\delta E_{s}^{m}=\gamma_{s}\left(A_{s}^{m+1}-A_{s}^{m}\right)$. As a final point, the potential variation $\delta \phi^{m}=\delta E_{s}^{m}+\delta E_{v}^{m}$ at step $m$ induced by elastic deformation can be calculated in order to determine the probability $P\left(\delta \phi^{m}\right)$, defined by eq. (4), that the system configuration will change.

- the second one concerns relaxation of the distortion elastic energy generated during the previous route, which involves no pressure variation. This is done by imposing small variation $\delta \varepsilon_{D}^{m}$ of the deviatoric part of the strain tensor $\varepsilon^{m}$. It allows the calculation of the potential variation $\delta \phi^{m}=\delta E_{\varepsilon_{D}}^{m}$

for the estimate of configuration change with respect to $P\left(\delta \phi^{m}\right)$. The function of the second route was shown to induce mass transport in relation to stress gradient [9]. To differentiate mass transport properties between the solid and vapor phases, the location of the interface within the Eulerian grid is required. Thus, a specific variable $C$, the phase function, is introduced. $C$ equals 1 in the solid phase and 0 in the vapor phase. The interface location is defined by $\mathrm{C}=0.5$.

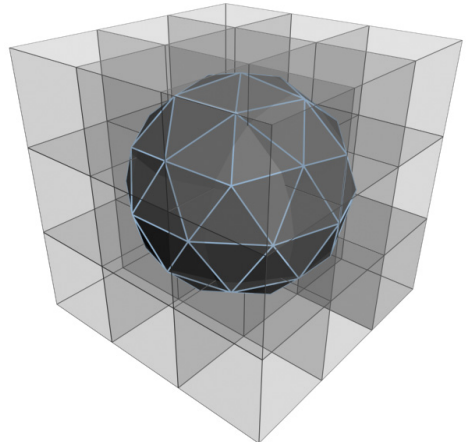

(a)

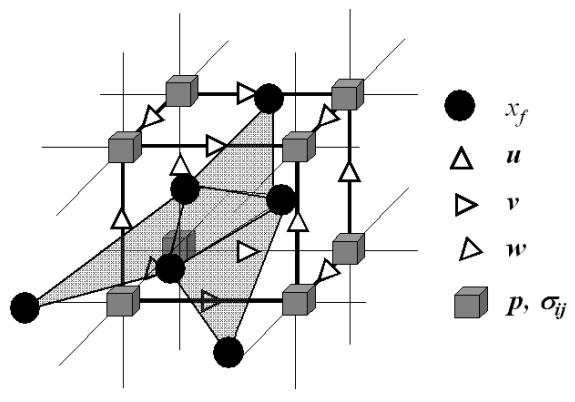

(b)

Figure 1: (a) The interface of the solid phase is discretized using a triangular mesh and is placed within a fixed Cartesian grid; (b) detailed visualization of the interfaced marker discretisation and of the staggered mesh used for the Monte Carlo calculation of the volume energy. $\nabla, \Delta, D$, vectors parallel to the X,Y,Z-axis; $\square$ scalar points. 


\section{Validation for the single spherical particle of the 3D implementation}

The Monte Carlo calculations have been performed using the following values of elastic constants for the two volume phases $\left(E_{Y}=4 \times 10^{6} \mathrm{~Pa}, v=0\right)$, surface tension $\left(\gamma_{s}=2.5 \times 10^{-3} \mathrm{~N} / \mathrm{m}\right)$ and temperature $(T=1 \mathrm{~K})$. The initial strain tensor within the two phases was $\varepsilon^{0}=0$. The spherical particle having a radius value $R=2.5 \mathrm{~mm}$ is centred within the vapor phase limited to a cell of $10 \times 10 \times 10 \mathrm{~mm}^{3}$. At the limit of the cell imposed displacements equal zero, insuring during minimisation the condition of a closed system in which the $\phi$-potential is defined.

The minimisation of the $\phi$-potential (eq. (1)) can be followed through the evolutions of $p_{1}$ and $p_{0}$, the mean pressures within the particle and the vapor phases, respectively (fig. 2). The equilibrium state corresponds to the large plateau at the end of the curves. The pressures within the two phases are constant and homogeneous as it is shown in fig. 2 with the $3 \mathrm{D}$ visualisation of an equatorial section of the particle.

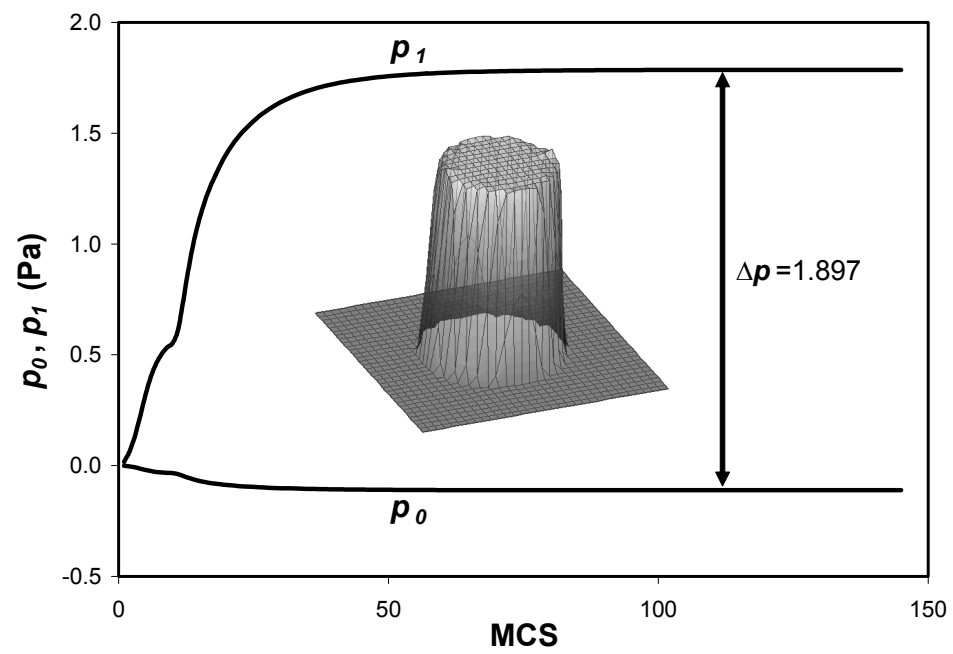

Figure 2: Curves: Evolution during the Monte Carlo minimisation $(\mathrm{MCS}=$ Monte Carlo Steps) of the mean value of the pressure $p_{1}$ within the spherical particle and $p_{0}$ within vapour for the $32 \times 32 \times 32$ grid. Centre: 3D visualisation of the pressure difference $\Delta p=1.897$ between the two phases on an equatorial plane of the particle.

To analyse the accuracy of the Monte Carlo methodology in calculating the stress response to interfacial forces, we have compared (for the final equilibrium state) the pressure difference between the two phases $\Delta p_{N U M}=\left(p_{1}-p_{0}\right)$ with the exact solution given by Laplace's formula $\Delta p_{t h}=2 \gamma_{s} / R_{N U M}$, where $R_{N U M}$ is the numerical cylinder radius calculated from the Eulerian volume of the phase 
function C. In fig. 3 , the relative error $\left(\Delta p_{N U M}-\Delta p_{t h}\right) / \Delta p_{t h}$ versus the number of scalar nodes of the mesh grid axis $N$ is plotted. This relative error is compared to that obtained with the Front Tracking approach [13]. The comparison analysis clearly shows that both methods, Monte Carlo and Front Tracking, have first order spatial convergences. A better accuracy is nevertheless obtained with the Monte Carlo model, which corresponds, for similar mesh size, to a relative error twice lower.

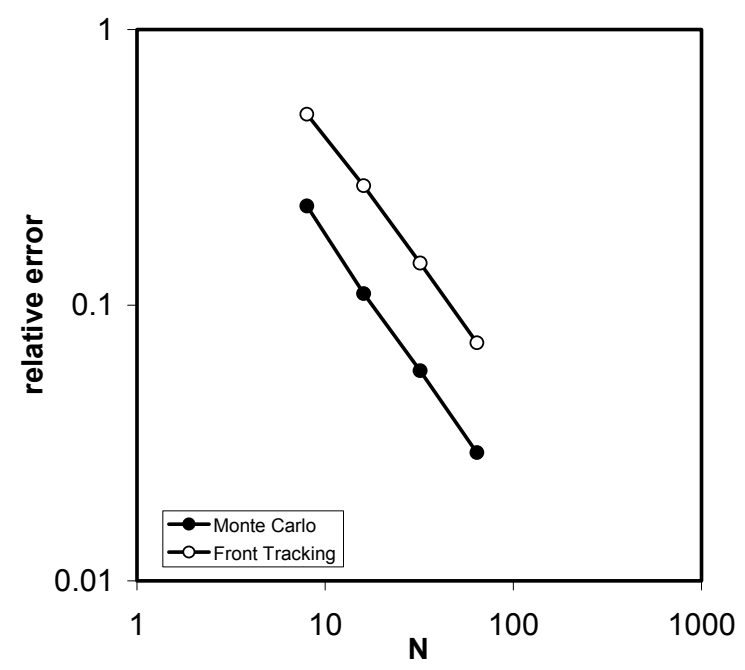

Figure 3: The Monte Carlo relative error $\left(\Delta p_{N U M}-\Delta p_{t h}\right) / \Delta p_{t h}$ for the pressure difference $\Delta p_{N U M}=\left(p_{1}-p_{0}\right)$ between the two phases with respect to Laplace's law $\Delta p_{t h}=\gamma_{s} / R_{N U M}\left(R_{N U M}\right.$ being the radius of the phase function $\mathrm{C}$ ), is plotted versus the scalar nodes of the mesh grid axis $N$ and compared for equivalent criterion with the relative error obtained from the front tracking method [13].

\section{Modelling of a real arrangement of particles extracted from microtomography image}

In order to show our progress towards real material systems modelling, we have performed calculations on a four-particle system (fig. 4(b)) extracted from a 3D image of a soda-lime glass sample (fig 4(a)) obtained by X-ray computed microtomography [14]. The microtomographic acquisitions were performed at the European Synchrotron Radiation Facility (ESRF, Grenoble, France) site on the ID19 imaging and diffraction beam line. The holes on the extracted particle surface corresponding to the contact between other surrounding particles have been closed for calculation feasibility. The surface tension considered for calculations are that of the soda-lime glass material [15] i.e. $\gamma_{s}=0.36 \mathrm{~J} / \mathrm{m}^{2}$. The 
elastic constants considered for the Monte Carlo calculation $\left(E_{Y}=4 \times 10^{6} \mathrm{~Pa}, v=0\right)$ are the same for the solid and vapour phases and thus have no physical meaning in the present case. As it can be seen on the micro tomographic image (fig. 4(a)), the glass material was characterised by a regular grain size centred around a radius of $60 \mu \mathrm{m}$.

The Navier Stokes resolution [16] of the microstructure evolution during sintering using the Front Tracking approach is presented in figure 5. The increase of the contact radius between particles until the contact coalescence as well as the pore closing is successfully modelled. This result highlights the numerical possibility of the coupled Eulerian/Lagrangian discretisation (fig. 1) to deal with 3D interface movements and disappearances. Nevertheless, the relevance of the calculated sintering kinetics will depend on the accuracy of the surface tension induced pressure within particles which is the driving force for mass transport. Unless very fine grids are implemented, this accuracy is difficult to reach on Eulerian grids when very small interface curvatures are involved, as it is the case at the contact zone for early sintering times. To discuss about that point, the Navier Stokes and Monte Carlo pressure fields obtained for two sections of the initial particle configuration are compared in fig. 6. One can notice that important pressure variations are located at the contact zones going from tensile values in that zone to compressive values in the direction of the outer lobe of the particles. The average value of pressure difference $\left(p_{1}-p_{0}\right)=1.1 \times 10^{4} \mathrm{~Pa}$ between the solid and the vapour phases at the outer lobe of the particles interface is very close to that of Laplace's law $2 \gamma_{\mathrm{s}} / a=1.2 \times 10^{4} \mathrm{~Pa}$, considering the mean value of the particle radius $a=60 \mu \mathrm{m}$.

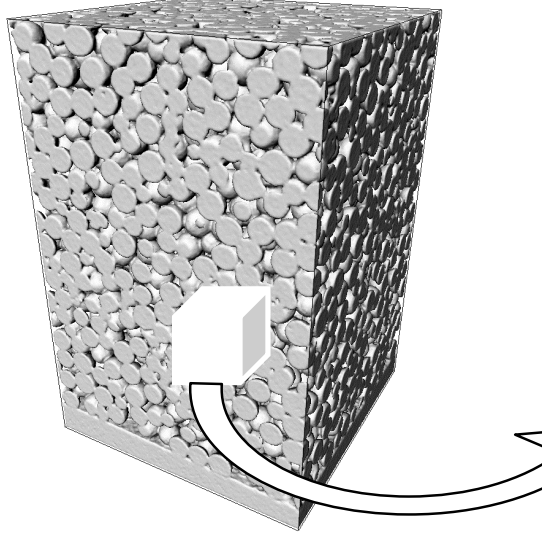

(a)

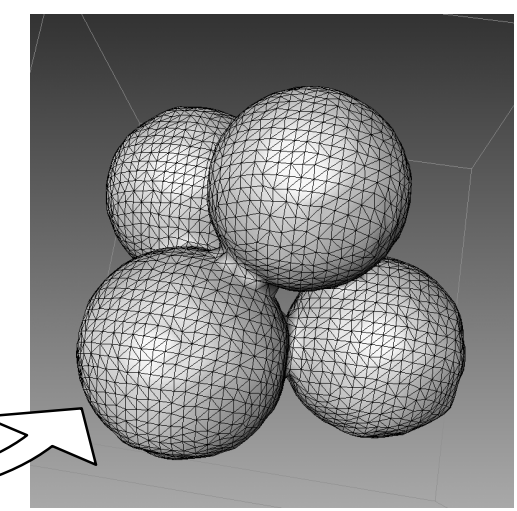

(b)

Figure 4: (a) X-ray computed microtomography image of a glass particle compact performed at European Synchrotron Facility (ESRF, Grenoble, France) [14]. The glass material was characterised by a regular grain size of $60 \mu \mathrm{m}$-radius; (b) four-particle system extracted from the powder compact for Monte Carlo and Navier Stokes calculations. 


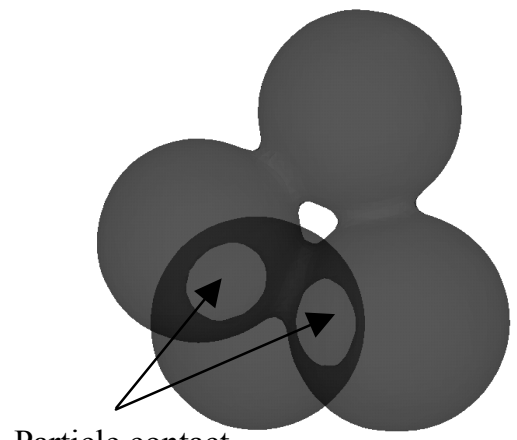

Particle contact

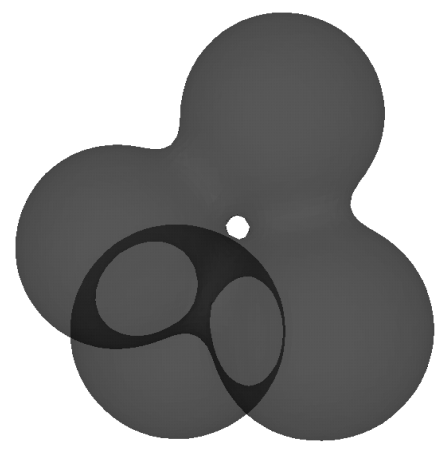

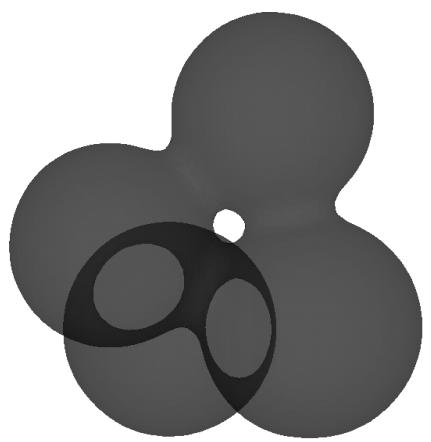

Particle contact

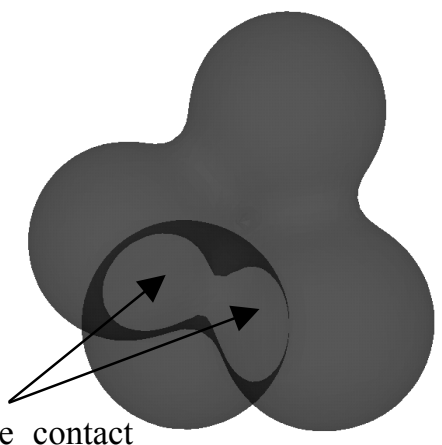

coalescence

Figure 5: Navier-Stokes 3D evolution of the four-particle morphology during the sintering process showing pore closing and particle contact coalescence.

Now, the comparative analysis of the Monte Carlo and Navier Stokes results shows that the two tensile pressure peaks are clearly present in the three contact zones presented in fig 6 for the Monte Carlo simulation. They are sharp and located precisely just below the interface. The pressure gradient is regularly distributed in agreement with the Monte Carlo previous results based on the 2D Lagrangian volume discretisation. On the opposite, the Navier Stokes simulation leads in some cases to a unique broaden tensile peaks centred within the contact zone which are associated to no well-drawn pressure gradient distribution. On the basis of these results, the Monte Carlo calculation of the surface tension induced pressure, which results from potential minimisation involving displacement of the Lagrangian nodes, is shown to be more accurate than a method resulting from interface curvature calculation after projection on a structured grid non-conforming to the interface. The Front Tracking method thus induces an inaccurate macroscopic interpretation of the small-scale structure of interface, resulting from a simulation on a too coarse Eulerian grid. 

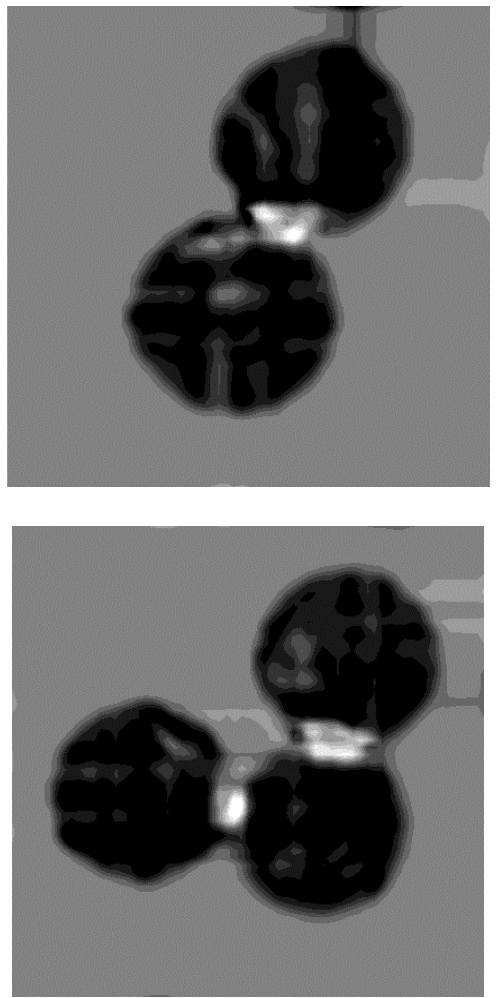

A
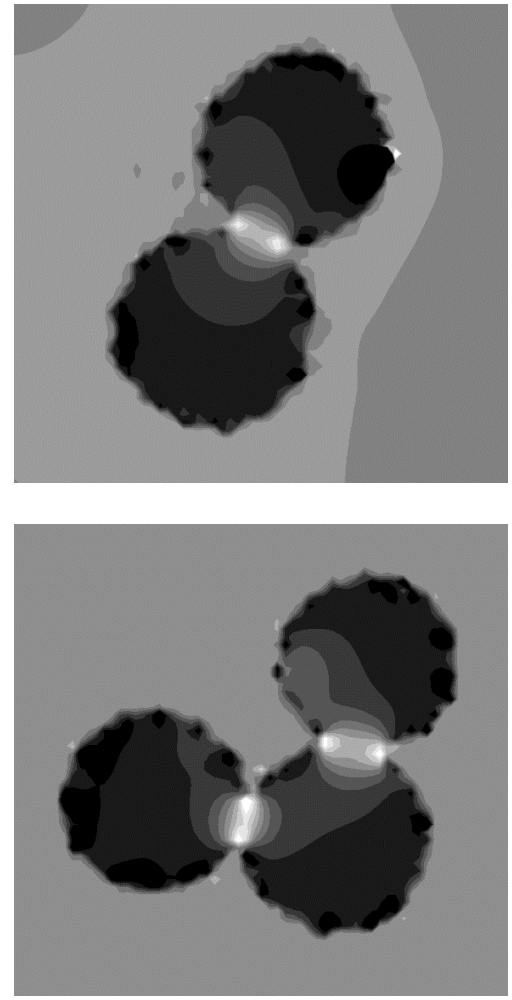

B

Figure 6: Comparison between Navier Stokes (column A) and Monte Carlo (column B) resolution for a $64 \times 64 \times 64$ grid of the hydrostatic pressure map within two sections of the four particle system. The pressure scale is $p_{1}=-10^{4} \mathrm{~Pa}$ for white colour to $p_{1}=10^{4}$ for black colour.

\section{Conclusion}

For the first time, the Monte Carlo numerical modelling dedicated to sintering processes have been implemented in an Eulerian fixed Cartesian grid framework in order to deal with 3D microstructures, the interface being tracked thanks to a Lagrangian grid. As a first step, the Monte Carlo simulations have been validated by comparisons to Laplace's law. A first order spatial convergence, two times more accurate that the fluid mechanics Eulerian Front Tracking method, has been obtained. As a second step, the calculation of the pressure gradient within a four particle arrangement extracted from a 3D image of a real glass powder compact was shown to be accurately estimated even at the particle contact zone where low surface curvatures are involved. Our objective now is to couple stochastic 
Monte Carlo and deterministic Navier Stokes approaches in order to obtain sintering kinetics as precise as possible to be compared with experimental data from microtomography.

\section{References}

[1] J. Pan, H.N. Ch'ng, A.C.F. Cocks, Mech. Mater. 37 705-721 (2005).

[2] Y.U. Wang, Acta Mater. 54 943-961 (2006).

[3] E.A. Olevsky, V. Tikare, T. Garino, J. Am. Ceram. Soc. 89 [6] 1914-1922 (2006).

[4] H. Matsubara, J. Ceram. Soc. Japan, 113 [4] 263-268 (2005)

[5] Y. Wang, L.Q; Chen, in Methods and Material Research, Wiley, New York (2000).

[6] A. Jagota, and P. R. Dawson, J. Am. Ceram. Soc., 73 [1] 173-77 (1990).

[7] G.A.L. van de Vorst, Eng. Anal. Boundary Elem., 14 193-207 (1994).

[8] H. Zhou and J. J. Derby, J. Am. Ceram. Soc., 81 [3] 533-40 (1998).

[9] S. Bordère, D. Gendron, J. M. Heintz and D. Bernard, J. Am. Ceram. Soc., 88 [8] 2071-2078 (2005).

[10] S. Bordère, D. Gendron, and D. Bernard, Scripta Mater., 55 267-270 (2006)

[11] S. Bordère, Scripta Mater., 55 879-882 (2006)

[12] S. Bordère, S. Vincent, J.P. Caltagirone, submitted to Computers and Fluids

[13] S. Shin and D. Juric, J. Comput. Phys., 180 427-470 (2002).

[14] D. Bernard, D. Gendron, J.-M. Heintz, S. Bordère, J. Etourneau, Acta Mater., 53 121-128 (2005).

[15] H. Scholze, Le verre: Nature, Structure et Propriétés, second ed., Institut du verre, Paris, 1981.

[16] S. Vincent and J.-P. Caltagirone, J. Compu. Phys., 169 172-215 (2000) 